\title{
A MEAN VALUE FORMULA FOR THE SPIN GROUP
}

\section{LAWRENCE VERNER ${ }^{1}$}

ABSTRACT. An adelic mean value formula is proved for two-connected algebraic homogeneous spaces, generalizing Siegel's formula in the geometry of numbers. The case of the spin group acting on the generalized sphere furnishes an example. The procedure consists in applying Galois cohomological techniques to the method of Ono.

Let $q$ be a quadratic form on $\mathbf{C}^{n}$, and let $X=\left\{x \in \mathbf{C}^{n}: q(x)=1\right\}$. The group $S O(q)=\{g \in \mathrm{SL}(n, \mathrm{C}): q(g x)=q(x)\}$ acts transitively on $X$. Applying Witt's theorem to the homogeneous space $(S O(q), X)$, Ono proved a "mean value formula" which is analogous to Siegel's mean value theorem [4]. Our intention is to prove the mean value formula for certain other spaces.

The action of $S O(q)$ on $X$ can be lifted to $\operatorname{Spin}(q)$, the universal covering group of $S O(q)$, as follows. Let $p: \operatorname{Spin}(q) \rightarrow S O(q)$ be the covering map. For $g \in \operatorname{Spin}(q), x \in X$, set $g^{*} x=p(g) x$. Then Spin $(q)$ acts transitively on $X$ but does not satisfy the Witt condition unless $q$ has maximal Witt index. In general we consider an algebraic group $G$ acting transitively on a variety $X$. Ono has shown that the mean value theorem holds for $(G, X)$ if certain topological conditions are satisfied [3]. A variation of Ono's methods will enable us to prove a theorem which contains the mean value formula for the spin group as a special case.

1. Statement of the Theorem. Let $G$ be a connected linear algebraic group defined over a field $k$. Let $X$ be an algebraic variety defined over $k$ upon which $G$ acts $k$-rationally. The pair $(G, X)$ is a homogeneous space defined over $k$ if the action is transitive and $X_{k} \neq \varnothing$.

Theorem. Let $(G, X)$ be a homogeneous space defined over an algebraic number field $k$. Suppose $X$ is 2-connected as a complex manifold and $G$ is

Received by the editors May 3, 1974.

AMS (MOS) subject classifications (1970). Primary 12A85; Secondary 12B20.

Key words and phrases. Homogeneous space, quadratic form, algebraic group, Tamagawa number.

1 This paper describes a part of my Ph.D. thesis. I would like to thank Professor Takashi Ono for his assistance. 
1-connected as a complex Lie group. Then, for every continuous function $f$ on the adele space $X_{A}$ with compact support,

$$
\int_{G_{A} / G_{k}} \sum_{x \in X_{k}} f(g x) d \dot{g}=\int_{X_{A}} f(x) d x .
$$

In particular, both sides of the formula are meaningful and finite.

If $n \geq 3$, the group Spin $(q)$ is 1-connected, and if $n \geq 4$, the "sphere" $X$ is 2-connected (see [3, Exercise II, p. 279]). If $n \geq 5$, then by Hasse's theorem $q(x)=1$ has a rational solution and so $(\operatorname{Spin}(q), X)$ is a homogeneous space defined over $\mathbf{Q}$ satisfying the conditions of the Theorem.

Our proof of the Theorem requires the conjectures of Weil and Kneser on 1-connected groups, although for the spin group these conjectures are known results.

2. Canonical measures. If $G$ is an algebraic group with a finite fundamental group, then there is a canonical Haar measure $d g$ of the adele group $G_{A}$, and the measure of the quotient space $\tau(G)=\int G_{A} / G_{k} d \dot{g}$ is finite (see [5]). Canonical measures can also be defined on suitable homogeneous spaces.

Lemma 1. If $(G, X)$ satisfies the conditions of the Theorem, then the isotropy subgroup $G_{x}=\{g \in G: g x=x\}$, is 1-connected for each $x \in X$.

Proof. Fix $x \in X$. The map $G \rightarrow X$, given by $g \rightarrow g x$, gives rise to an exact sequence.

$$
0 \rightarrow G_{x} \rightarrow G \rightarrow X \rightarrow 0
$$

This in turn induces a finite covering

$$
0 \rightarrow G_{x} /\left(G_{x}\right)_{0} \rightarrow G /\left(G_{x}\right)_{0} \rightarrow X \rightarrow 0
$$

where $\left(G_{x}\right)_{0}$ denotes the connected component of the identity in $G_{x}$. Since $X$ is simply-connected, the covering must be trivial; hence, $G_{x} /\left(G_{x}\right)_{0}=0$, i.e., $G_{x}$ is connected.

By the homotopy exact sequence associated with (E) we have $\pi_{2}(X) \rightarrow$ $\pi_{1}\left(G_{x}\right) \rightarrow \pi_{1}(G)$. Our hypotheses imply that $0=\pi_{2}(X)=\pi_{1}(G)$, and so $G_{x}$ is simply-connected.

Proposition. If $(G, X)$ is a homogeneous space defined over $k$, satisfying the conditions of the Theorem, then

(a) $X_{k}$ is discrete in $X_{A}$; 
(b) there is a canonical measure $d x$ on $X_{A}$;

(c) $d x$ is $G_{A}$-invariant.

Proof. Lemma 1 shows that $(G, X)$ is a "special homogeneous space" in the language of [3]. Theorem 4.1 of [3] therefore applies.

Weil's conjecture states that if $G$ is a 1-connected algebraic group, then $\tau(G)=1$. Consequently our Theorem gives a formula for the mean value of the function $g \mapsto \Sigma_{x \in X_{k}} f(g x)$.

3. Galois cohomology. Let $(G, X)$ be a homogeneous space defined over a field $k$. Although $G$ acts transitively on $X, G_{k}$ need not act transitively on $X_{k}$. Instead, we have, for each $\xi \in X_{k}$, an exact sequence of Galois cohomology sets:

$$
0 \longrightarrow G_{\xi, k} \stackrel{\iota_{k}}{\longrightarrow} G_{k} \stackrel{\kappa_{k}}{\longrightarrow} X_{k} \stackrel{\delta_{k}}{\longrightarrow} H^{1}\left(k, G_{\xi}\right) \stackrel{\Delta_{k}}{\longrightarrow} H^{1}(k, G) .
$$

Two elements in $X_{k}$ have the same image in $H^{1}\left(k, G_{\xi}\right)$ if and only if they are in the same $G_{k}$-orbit. The main properties of Galois cohomology can be found in [1].

Let $G$ be an algebraic group defined over an algebraic number field $k$. The completions $k \rightarrow k_{v}$ induce a map

$$
a: H^{1}(k, G) \rightarrow \Pi H^{1}\left(k_{v}, G\right),
$$

the product being taken over all places of $k$. Kneser has conjectured that $\alpha$ is bijective if $G$ is 1-connected [2]. This is known to hold for all groups without an $E_{8}$-factor. In what follows we assume the validity of Kneser's conjecture.

Lemma 2. Let $(G, X)$ satisfy the conditions of the Theorem. Then, $G_{A} X_{k}=X_{A}$.

Proof. Let $x \in X_{A}$, and consider the commutative diagram:

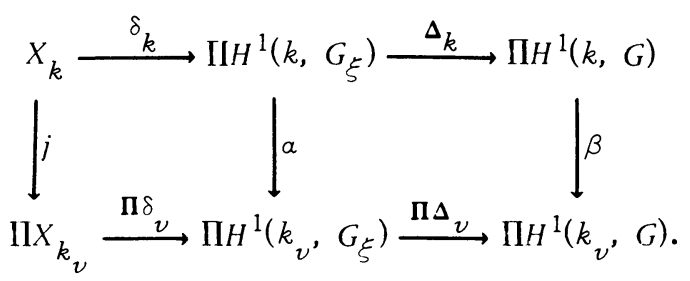

We view $x \in \Pi X_{k_{v}}$. Let $\left(c_{v}\right)=\Pi \delta_{v}(x)$. Lemma 1 shows that $G_{\xi}$ is 1 -connected and so by Kneser's conjecture $\alpha$ is surjective and we can lift $\left(c_{v}\right)$ to $c \in H^{1}\left(k, G_{\xi}\right)$. Now 


$$
\beta \circ \Delta_{k}(c)=\Pi \Delta_{v} \circ \alpha(c)=\Pi \Delta_{v}\left(c_{v}\right)=\Pi \Delta_{v} \circ \operatorname{II} \delta_{v}(x)=0 .
$$

By Kneser's conjecture, $\beta$ is injective and, hence, $\Delta_{k}(c)=0$. By exactness we can write $c=\delta_{k}(\eta)$, for some $\eta \in X_{k}$. Then

$$
\Pi \delta_{v} \circ j(\eta)=\alpha \circ \delta_{k}(\eta)=\alpha(c)=\left(c_{v}\right)=\Pi \amalg \delta_{v}(x)
$$

i.e., for each place $v, \delta_{v}(\eta)=\delta_{v}(x)$, and so $x$ and $\eta$ are in the same orbit under $G_{k_{v}}$. For each $v$ select $g_{v}^{\prime} \in G_{k_{v}}$ such that $g_{v}^{\prime} \eta=x$. For almost all places $v$, the group of $v$-integral points, $G_{0}$, acts transitively on $X_{0}$ (see [1, p. 161]). Let $S$ be the set of places where this transitivity fails. For each $v \notin S$ choose $h_{v} \in G_{0}{ }_{v}$ such that $h_{v} \eta=x$. Now set

$$
g_{v}= \begin{cases}g_{v}^{\prime} & \text { if } v \in S, \\ b_{v} & \text { if } v \notin S .\end{cases}
$$

Then $g=\left(g_{v}\right) \in G_{A}$, and $x=g \eta \in G_{A} X_{k}$ as desired.

Lemma 3. If $(G, X)$ satisfies the conditions of the Theorem, then the map $J: G_{k} \backslash X_{k} \rightarrow G_{A} \backslash X_{A}$, given by $J\left(G_{k} \xi\right)=G_{A} \xi$, is bijective.

Proof. Take $G_{A} x \in G_{A} \backslash X_{A}$. By Lemma 2 we can write $x=g \eta$, with $g \in G_{A}, \eta \in X_{k}$. Then $G_{A} x=G_{A} \eta=J\left(G_{k} \eta\right)$, so $J$ is surjective. To see that $J$ is injective consider the commutative diagram

$$
\begin{aligned}
& G_{k} \stackrel{\kappa_{k}}{\longrightarrow} X_{k} \stackrel{\delta_{k}}{\longrightarrow} H^{1}\left(k, G_{\xi}\right) \\
& \downarrow j_{G} \quad j_{X} \quad \downarrow a \\
& \Pi G_{k} \stackrel{\Pi \kappa_{v}}{\longrightarrow} \Pi X_{k} \stackrel{\Pi \delta_{v}}{\longrightarrow} \Pi H^{1}\left(k_{v}, G_{\xi}\right) \text {. } \\
& J\left(G_{k} \xi\right)=J\left(G_{k} \eta\right) \quad\left(\left(\xi, \eta \in X_{k}\right) \Rightarrow \eta \in G_{A} \xi\right) \\
& \Rightarrow j_{X}(\eta) \in \operatorname{Im}\left(\Pi \kappa_{v}\right)=\operatorname{Ker}\left(\Pi \delta_{v}\right) \\
& \Rightarrow \alpha \circ \delta_{k}(\eta)=0 \\
& \Rightarrow \delta_{k}(\eta)=0 \quad(\text { Kneser's conjecture }) \\
& \Rightarrow \eta \in \operatorname{Im}\left(\kappa_{k}\right)=G_{k} \xi \Rightarrow G_{k} \xi=G_{k} \eta \text {. }
\end{aligned}
$$

4. Proof of the Theorem. Let $(G, X)$ be a homogeneous space defined over $k$, satisfying the conditions of the Theorem. Let $f$ be a continuous 
function on $X_{A}$ with compact support. For each $\xi \in X_{k}$ let $d u_{\xi}$ be the canonical Haar measure on $G_{\xi, A^{*}}$

Lemma 4 [3, Lemma 5.1]. Each orbit $G_{A} \xi$ is open in $X_{A^{*}}$.

Lemma 4 shows that the measure $d x$ on $X_{A}$ restricts to a measure on $G_{A}$.

As an application of Fubini's theorem we have (see [5, Lemma 2.4.2])

$$
\int_{G_{A} / G_{k}} \sum_{x \in G_{k} \xi} f(g x) d \dot{g}=\int_{G_{A} \xi} f(x) d x \cdot \int_{G_{\xi, A} / G_{\xi, k}} d \dot{u}_{\xi}=\int_{G_{A} \xi} f(x) d x,
$$

by Weil's conjecture applied to $G_{\xi}$.

Let $D$ be a fundamental domain for $G_{k}$ in $X_{k}$. Lemma 3 shows that $D$ is also a fundamental domain for $G_{A}$ in $X_{A}$. Hence,

$$
\begin{aligned}
\int_{G_{A} / G_{k}} \sum_{x \in X_{k}} f(g x) d \dot{g} & =\int_{G_{A} / G_{k}} \sum_{\xi \in D} \sum_{x \in G_{k} \xi} f(g x) d \dot{g} \\
& =\sum_{\xi \in D} \int_{G_{A} / G_{k}} \sum_{x \in G_{k} \xi} f(g x) d \dot{g} \\
& =\sum_{\xi \in D} \int_{G_{A} \xi} f(x) d x=\int_{X_{A}} f(x) d x=\int_{X_{A}} f(x) d x .
\end{aligned}
$$

\section{REFERENCES}

1. A. Borel and J.-P. Serre, Théorèmes de finitude en cohomologie galoisienne, Comment. Math. Helv. 39 (1964), 111-164. MR 31 \#5870.

2. M. Kneser, Hasse principle for $H^{1}$ of simply connected groups, Proc. Sympos. Pure Math., vol. 9, Amer. Math. Soc., Providence, R. I., 1966, pp. 159-163. MR 36 \# 3788.

3. T. Ono, A mean value theorem in adele geometry, J. Math. Soc. Japan 20 (1968), 275-288. MR $37 \# 6286$.

4. C. L. Siegel, A mean value theorem in geometry of numbers, Ann. of Math. (2) 46 ( 1945), 340-347.

5. A. Weil, Adeles and algebraic groups, Institute for Advanced Study, Princeton, N. J., 1966. MR 6, 257.

DEPARTMENT OF MATHEMATICS, CITY UNIVERSITY OF NEW YORK, BARUCH COLLEGE, NEW YORK, NEW YORK 10010 